"This is the peer reviewed version of the following article: [Lovell, M., Luckett, T., Boyle, F., Stubbs, J., Phillips, J., Davidson, P. M., Olver, I., von Dincklage, J. and Agar, M. (2015), Adaptation of international guidelines on assessment and management of cancer pain for the Australian context. Asia-Pac $J$ Clin Oncol, 11: 170-177] which has been published in final form at [ http://dx.doi.org/10.1111/ajco.12352 ]. This article may be used for non-commercial purposes in accordance with Wiley Terms and Conditions for Self-Archiving." 


\section{Adaptation of international guidelines on assessment and management of cancer pain for the Australian context}

\section{Authors}

Melanie Lovell, ${ }^{1,2}$ Tim Luckett, ${ }^{3,4,5}$ Frances Boyle, ${ }^{2,6}$ John Stubbs, ${ }^{7}$ Jane Phillips, ${ }^{4}$ Patricia Mary Davidson, ${ }^{4,8}$ Ian Olver, ${ }^{9}$ Jutta von Dincklage, ${ }^{9}$ Meera Agar ${ }^{1,5,10}$

\section{Address of institutions}

1. HammondCare, Sydney, New South Wales (NSW), Australia

2. Sydney Medical School, The University of Sydney, Sydney, NSW, Australia

3. Improving Palliative Care through Clinical Trials (ImPaCCT), Sydney, NSW, Australia

4. Centre for Cardiovascular and Chronic Care, University of Technology Sydney (UTS), Faculty of Health, Sydney, NSW, Australia

5. South Western Sydney Clinical School, University of New South Wales (UNSW), Sydney, NSW, Australia

6. Patricia Ritchie Centre for Cancer Care and Research, Mater Hospital, Sydney, NSW, Australia

7. CanSpeak, Sydney, NSW, Australia

8. School of Nursing, Johns Hopkins University, Baltimore, Maryland, USA

9. Cancer Council Australia, Sydney, NSW, Australia

10. Ingham Institute of Applied Medical Research, Sydney, NSW, Australia

\section{Correspondence}

Dr Melanie Lovell

Greenwich Hospital, 97-115 River Road, Greenwich, NSW 2065, Australia

E: mlovell@hammond.com.au

P: +612 87883953

F: +61299038383 


\section{Abstract}

\section{Aim}

To develop clinical practice guidelines for screening, assessing and managing cancer pain in Australian adults.

\section{Methods}

This three phase project utilised the ADAPTE approach to adapt international cancer pain guidelines for the Australian setting. A Working Party was established to define scope, screen guidelines for adaptation, and develop recommendations to support better cancer pain control through screening, assessment, pharmacological and non-pharmacological management, and patient education.

Recommendations with limited evidence were referred to Expert Panels for advice before the draft guidelines were opened for public consultation via the Cancer Council Australia Cancer Guidelines Wiki platform in late 2012. All comments were reviewed by the Working Party and the guidelines revised accordingly.

\section{Results}

Screening resulted in six international guidelines being included for adaptation - those developed by the Scottish Intercollegiate Guidelines Network (2008), National Health Service Quality Improvement Scotland (2009), National Comprehensive Cancer Network (2012), European Society of Medical Oncology (2011), European Association for Palliative Care (2011, 2012) and National Institute of Clinical Excellence (2012). Guideline adaptation resulted in 55 final recommendations The guidelines were officially launched in November 2013.

\section{Conclusion}

International guidelines can be efficiently reconfigured for local contexts using the ADAPTE approach. Availability of the guidelines via the Cancer Council Australia Wiki is intended to promote uptake and enable recommendations to be kept up to date. Resources to support implementation will also be made available via the Wiki if found to be effective by a randomised controlled trial commencing in 2015.

\section{Keywords}

Guideline, Practice guideline, Pain, Cancer, Australia 


\section{Background}

Pain is experienced by up to $75 \%$ of people with cancer and rated as moderate-to-severe by $40-50 \%$ and severe by $25-30 \% .{ }^{1}$ As the population ages, other comorbid conditions (e.g. osteoarthritis) are increasingly contributing to the complexity and challenges of cancer pain management. ${ }^{2}$ Despite the prevalence of pain in people with cancer, it is under-identified and under-treated internationally. ${ }^{3}$ In Australia, cancer pain has been identified as a priority area for improvement at national and state levels. ${ }^{4,5}$ There is evidence that implementation of evidence-based guidelines can improve quality of care and outcomes for cancer pain. ${ }^{6}$ However, implementing guidelines is not straightforward, with barriers frequently encountered across the levels of health system, clinician and patient. ${ }^{7}$ In 2010 , the Australian National Pain Summit identified that the promotion of guidelines and strategies to support implementation would be required to improve cancer pain management and outcomes nationally. ${ }^{8}$

To better understand current practices and clinician needs, a national online survey was undertaken from August 2011 to April 2012. Five hundred and thirty seven health professionals responded from across most disciplines involved in cancer care. ${ }^{9-11}$ Less than half $(46 \%)$ of respondents reported using guidelines for managing cancer pain -likely to be an over-estimate of the national picture given the 'volunteer effect' expected for open surveys. Ninety per cent were supportive of new Australian cancer pain guidelines and implementation strategies, but there was support for these to build on existing international guidelines rather than for recommendations to be developed de novo. Guideline contents identified as potentially useful by more than $90 \%$ of respondents included: advice on best practice for specific cases of cancer pain (e.g. neuropathic), guidance on assessing patient-identified priorities, and inclusion of patient 'action plans' to aid self-management.

In this paper, we describe the methods used to adapt international guidelines for the Australian setting and discuss current work aimed at supporting their implementation.

\section{Method}

An Organizing Committee was formed to plan and oversee the project. The Committee included the Chair of the Working Group from the National Pain Summit (ML), as well as an implementation scientist (PMD) and palliative care physician (MA) with previous experience in guideline development, and coordination (TL) and administrative (AG) support. The Committee lodged its 
intention to develop guidelines for cancer pain on the National Health and Medical Research Council (NHMRC) online Guidelines in Development Register in May 2011.

The Organizing Committee chose the ADAPTE approach ${ }^{12}$ as the only formal method available and one endorsed by the Guidelines International Network (GIN). ADAPTE specifies a three phase process of Set-up, Adaptation and Finalization (Figure 1). A large number of guidelines have been developed using this process including those for cancer symptom management. ${ }^{13,14}$

\section{Figure 1}

During the Set-up phase, the Organizing Committee agreed that synthesis and adaptation would likely be required across a number of guidelines rather than a single candidate, and convened a Working Party to provide expert guidance. The Working Party comprised physicians and nurses in palliative care (ML, MA, JP) and medical oncology (FB), a consumer representative (JS) and coordinator (TL). It held its inaugural meeting in January 2012 and met five more times through 2012 and 2013. Disclosures of interests were declared by each member and updated as required at each meeting. Only FB declared a relevant interest, relating to her membership of the Advisory Board for Takeda Pharmaceuticals Australia Pty Ltd. A medical writer was engaged to assist with wording and formatting of recommendations to maximize clarity and ease of reading and minimize ambiguity.

During the Adaptation phase, discussions were initially aimed at more clearly defining the focal Population, Intervention, Professionals, Outcomes and Health setting (PIPOH) and identifying international guidelines suitable for adaptation, as well as Australian guidelines on related topics. The Working Party agreed that the target population should include adults with cancer of any type or stage. A primary focus on cancer-related pain was agreed to provide optimum scope for recommendations; existing Australian guidelines for acute pain meant no new recommendations were needed for this problem. ${ }^{15}$ Both pharmacological and non-pharmacological management of cancer pain were considered important topics for inclusion. Given the multi-disciplinary and crosssector nature of contemporary Australian cancer care, it was agreed that the guidelines should be aimed at a broad readership of clinicians from different disciplines working in primary, secondary, tertiary and residential settings, including health professionals not specializing in oncology or palliative care.

Existing guidelines were sought via the reference lists of previous reviews ${ }^{16-18}$ and searches of online databases and clearing houses identified by the ADAPTE manual. ${ }^{12}$ Guidelines were screened according to the following eight criteria, based on the agreed PIPOH and requirements for currency 
and evidence: 1) a primary focus on adults with chronic cancer pain; 2) relevance across tumor types and stages; 3 ) inclusion of recommendations for assessment and/or management of pain by means of either pharmacological or non-pharmacological intervention; 4) capacity to inform pain assessment and management across disciplines and settings; 5) published in the previous 3 years (i.e. 2008 or later); 6) national or international coverage (i.e., not center-specific); 7) availability in English; and 8 ) independently rated as 'recommended' or 'strongly recommended' by two members of the Working Party based on criteria of the Appraisal of Guidelines Research \& Evaluation (AGREE) Instrument. ${ }^{19}$ Developers of the original guidelines were contacted via email as required to request evidence tables and further information about their development processes.

Synthesis of source guidelines was undertaken via exhaustive tabulation of recommendations aimed at identifying areas of agreement, near agreement, or lack of consensus. The Working Party then met to prioritize recommendations according to level of agreement between source documents, perceived importance to clinical care, and relevance to the Australian setting. Throughout, the Working Party was guided by principles of holistic person-centered care, equity of access and a concern to minimize inappropriate prescribing, especially in elderly patients. Recommendations meeting criteria were either directly adopted or modified as necessary, with sources referenced as appropriate.

Recommendations for pharmacological pain management and management of adverse effects for which there was no consensus were referred to two panels of expert palliative care physicians. Questions were posed to each panelist independently via an online survey. Any inconsistencies among responses were resolved via subsequent email discussion.

Where relevant, the Working Party included reference to other Australian clinical practice guidelines for the management of specific clinical problems (e.g. psychosocial concerns). ${ }^{20}$ To ensure recommendations in the adapted guidelines were based on up-to-date evidence, non-systematic searches of PubMed were undertaken to identify systematic reviews and randomized controlled trials (RCTs) published more recently than the source guidelines. The Organizing Committee and Working Party responded to priorities identified by the survey of current practice by conducting two systematic reviews to identify patient priorities for care and optimal approaches to supporting selfmanagement. ${ }^{21,22}$ Recommendations developed in response to these additional sources, guidance by the Expert Panels and the Working Party's own clinical experience were distinguished from those adapted from existing guidelines by the term 'consensus' in the draft and final guidelines, with the source and/or rationale provided. Practical tips about medication availability and prescribing were included as practice points.

Throughout the guideline development process, the Working Party sought opportunities to disseminate the project and elicit information on stakeholder needs via local, state, national and 
international fora attended by health professionals and consumers. This included 2012 meetings of Palliative Care New South Wales, NPS MedicineWise, the International Psycho-Oncology Society (held in Brisbane), European Association for Palliative Care and Multinational Association for Supportive Care in Cancer, and 2012 and 2013 meetings of the Cancer Nurses Society of Australia, the Pharmaceutical Society of Australia, Palliative Care Australia and Australian Pain Society.

During the Finalization phase, stakeholder feedback on draft recommendations was sought via public consultation. Comments were sought via the Cancer Council Australia Cancer Guidelines Wiki platform ${ }^{23}$ from 26th November 2012 until 11th January 2013, in accordance with the minimum of 30 days stipulated by NHMRC standards for guideline development. ${ }^{24}$ Invitations to contribute to the consultation were circulated via the Working Party's networks as well as local, state and national organizations with an interest in caring for people with cancer pain. See Box 1 for a list of national organizations that circulated the invitation to their members.

Box 1. National organisations which circulated an invitation to their members to participate in the guideline public consultation

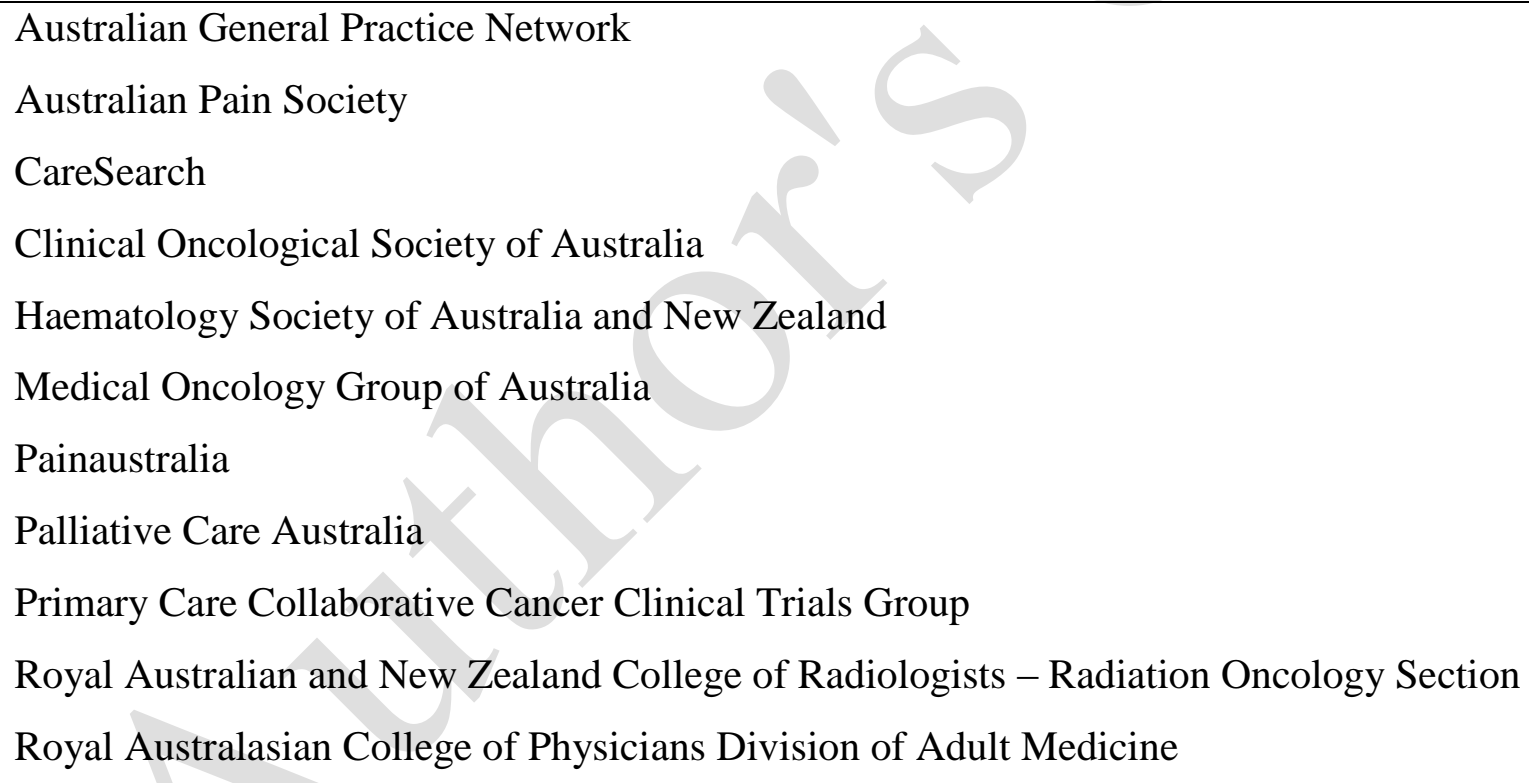

Plans for updating were agreed by the Working Party at meetings in late 2013 and early 2014. A decision was made to approach panels of experts on each section and seek their permission to be contacted every 3 months for advice on new evidence that should be considered for inclusion in updates. This approach was preferred to iterative systematic literature reviews not only because of the lesser resource requirements but also because it offered a means of disseminating the guidelines and building ownership amongst stakeholders. In addition, the Wiki platform enables guideline users to continue to submit comments and notification of new evidence on an ongoing basis. Inclusion of new evidence is assessed by the Working Party and a decision made whether to update the guidelines accordingly. 


\section{Results}

Evaluation against criteria resulted in six guidelines being included for adaptation (Table 1). It was agreed that no single guideline could be adopted in its entirety due to differences in practice internationally. Because the National Institute for Clinical Excellence (NICE) guideline ${ }^{25}$ was published only part-way through the adaptation process, its recommendations were used to inform later drafts rather than initial development. Guidelines offered by NICE and European Association of Palliative Care (EAPC) ${ }^{26}$ focused exclusively on pharmacological treatment.

Table 1. Six international guidelines on management of cancer pain included for adaptation to the Australian setting

\begin{tabular}{|c|c|c|c|}
\hline Organisation (Year) & Guideline title & Reference(s) & URL \\
\hline $\begin{array}{l}\text { Scottish Intercollegiate } \\
\text { Guidelines Network } \\
(\mathrm{SIGN})(2008)\end{array}$ & $\begin{array}{l}\text { Control of pain in adults } \\
\text { with cancer. A national } \\
\text { clinical guideline }\end{array}$ & 27 & $\begin{array}{l}\text { http://www.sign.ac.uk/pdf/SI } \\
\text { GN106.pdf }\end{array}$ \\
\hline $\begin{array}{l}\text { National Health Service } \\
\text { (NHS) Quality } \\
\text { Improvement Scotland } \\
\text { (2009) }\end{array}$ & $\begin{array}{l}\text { Best practice statement. The } \\
\text { management of pain in } \\
\text { patients with cancer. }\end{array}$ & 28 & $\begin{array}{l}\text { http://www.palliativecareguid } \\
\text { elines.scot.nhs.uk/documents/ } \\
\text { PAINCANCERREV_BPS_N } \\
\text { OV09.pdf }\end{array}$ \\
\hline $\begin{array}{l}\text { National Comprehensive } \\
\text { Cancer Network (NCCN) } \\
\text { (2012) }\end{array}$ & $\begin{array}{l}\text { NCCN clinical practice } \\
\text { guidelines in oncology. } \\
\text { Adult cancer pain. }\end{array}$ & 29,30 & http://www.nccn.org \\
\hline $\begin{array}{l}\text { European Society of } \\
\text { Medical Oncology } \\
\text { (ESMO) (2011) }\end{array}$ & Management of cancer pain & 31 & $\begin{array}{l}\text { http://annonc.oxfordjournals. } \\
\text { org/content/22/suppl_6/vi69.1 } \\
\text { ong }\end{array}$ \\
\hline $\begin{array}{l}\text { European Association for } \\
\text { Palliative Care (EAPC) } \\
\text { (2012) }\end{array}$ & $\begin{array}{l}\text { Use of opioid analgesics in } \\
\text { the treatment of cancer pain }\end{array}$ & $26 *$ & None \\
\hline $\begin{array}{l}\text { National Institute of } \\
\text { Clinical Excellence } \\
\text { (NICE) (2012) }\end{array}$ & $\begin{array}{l}\text { Opioids in palliative care: } \\
\text { safe and effective } \\
\text { prescribing of strong } \\
\text { opioids for pain in palliative } \\
\text { care of adults. }\end{array}$ & 25 & $\begin{array}{l}\text { http://www.nice.org.uk/nicem } \\
\text { edia/live/13745/59285/59285. } \\
\underline{\text { pdf }}\end{array}$ \\
\hline
\end{tabular}

* Informative systematic reviews have been published in a special issue (Issue 6) of Palliative Medicine, 2011 Volume 25. 
The Working Party noted that some recommendations from existing international guidelines might not be transferable to the Australian setting because of historical differences in clinical practice, drug availability and laws relating to opioid use. ${ }^{32}$ There was also concern to identify the best solutions to overcoming documented barriers to effective pain management. ${ }^{33}$ Recommendations featured in international guidelines but not considered transferable to the Australian setting included those from the UK's Scottish Intercollegiate Guidelines Network (SIGN), ${ }^{27}$ National Health Service (NHS) Quality Improvement Scotland ${ }^{28}$ and NICE ${ }^{25}$ guidelines relating to the World Health Organization (WHO) pain ladder, ${ }^{34}$ which the Working Party considered controversial in the Australian context, and the use of intravenous morphine recommended by NCCN which was not considered to be usual practice. $^{35}$

Recommendations from international guidelines and other sources were divided into sections of the Australian guidelines on patient-centered care, screening, assessment, self-management, pharmacological management and non-pharmacological management. The guideline adaptation process resulted in 55 final recommendations, two of which focused on patient-centered care, two on screening, one on assessment, three on self-management, 43 on pharmacological management and four on non-pharmacological management. The final recommendations and information about their sources are publically available on the Wiki. ${ }^{23}$ The Working Party agreed to prioritize recommendations supporting the essentials of care identified across international guidelines, namely: screening and assessment of pain, provision of regular and breakthrough analgesia, implementation of a bowel regimen for patients prescribed opioids, and patient education. ${ }^{36}$ It was agreed that a focus on these elements of care as key indicators of quality should be promoted by means of a simple clinical pathway that could be disseminated in hard copy for display in clinics

Public consultation resulted in 88 comments submitted via the Wiki for consideration by the Working Party, as well as 10 comments sent via email. Wiki comments and the Working Party's response to each can still be viewed under the relevant recommendations, which supports guideline transparency and, where applicable, a more detailed understanding of the rationale.

The guidelines were officially launched at the $40^{\text {th }}$ Annual Scientific Meeting of the Clinical Oncological Society of Australia, held in Adelaide in November 2013.

\section{Discussion}

Guidelines for assessing and managing cancer pain in adults have been developed for the Australian setting using the ADAPTE approach and are now available on the Cancer Council Australia Cancer Guidelines Wiki. ${ }^{23}$ This platform enables ongoing user feedback and rapid revision to ensure 
recommendations are up to date with emerging evidence. ${ }^{37}$ Users are invited to submit suggestions for revisions to the guidelines to the Working Party via the comments function available on the Wiki below each recommendation. The guidelines provide a point of care resource to support clinician assessment and management of adult cancer pain. The guidelines are not intended to replace expert care but rather to support the essentials of assessment and management by professionals less familiar with treating cancer pain.

Implementation of the guidelines will be supported by three strategies that have been shown to improve uptake of evidence-based care and overcome barriers in cancer pain management and other areas of patient care. ${ }^{6,38,39}$ These strategies are under development and will include patient selfmanagement resources, a program of health professional education and an audit tool to assess adherence to the essentials of cancer care as defined by the clinical pathway. Resources developed to support patient communication and self-management included templates to support goal setting, communication and monitoring of capacity to self-manage cancer pain and adequacy of support. These resources are intended to supplement an existing patient education booklet published by the Cancer Council New South Wales, ${ }^{40}$ which has proven efficacy in reducing pain intensity. ${ }^{41}$ Health professional education will be delivered via the QStream method (formally Spaced Education). QStream is an online learning format that has been shown to significantly improve knowledge and retention of guideline content in RCTs. ${ }^{42}$ Questions on the clinical content of guidelines are delivered via e-mail, with immediate feedback on selected answers and an opportunity to re-visit questions that were answered incorrectly. The audit tool will be based on a precedent developed by the Victorian Palliative Care Network ${ }^{43}$ and is currently being pilot tested alongside the patient-held resources at public and private palliative care and oncology sites in New South Wales. The tool assesses adherence to the key standards of screening, assessment, pharmacological management, management of constipation and patient education on which there is international consensus. ${ }^{36}$ It is hoped that health services will use these standards of care to evaluate their services and allocate resources.

An RCT will commence in 2015 to evaluate the ability of the guidelines and implementation resources to provide cost-effective improvements to cancer pain and related outcomes. Resources will be made available on the Wiki if found to be effective. 


\section{Acknowledgments}

We would like to acknowledge the contributions to this program by Jenni Harman for her work as medical writer on the guidelines and Natalie Marie and Mary-Rose Birch with regard to development of patient-held resources and pilot work.

HammondCare provided initial funds for the development of the guidelines.

The Cancer Institute New South Wales funds the salary of Dr Tim Luckett, who has provided coordination for the project.

Cancer Council Australia is providing the use of the Cancer Guidelines Wiki, which includes guideline hosting, maintenance and support services.

Development of implementation strategies and support from Anna Green has been supported by a competitive research grant from the University of Technology Sydney.

A pilot of the guidelines is underway with financial support from a competitive research grant from Pfizer Australia, which has no involvement in the research design or reporting.

\section{Disclosure of interests}

As noted in the manuscript, Prof Frances Boyle is a Member of Advisory Board for Takeda Pharmaceuticals Australia Pty Ltd. None of the other authors have any disclosures to make. 


\section{References}

1. van den Beuken-van Everdingen MHJ, de Rijke JM, Kessels AG, Schouten HC, van Kleef M, Patijn J. Prevalence of pain in patients with cancer: a systematic review of the past 40 years. Ann Oncol. 2007 September 1, 2007;18(9):1437-49.

2. Bender CM, Engberg SJ, Donovan HS, Cohen SM, Houze MP, Rosenzweig MQ, et al. Symptom clusters in adults with chronic health problems and cancer as a comorbidity. Oncology Nursing Forum. 2008 Jan;35(1):E1-E11.

3. Deandrea S, Montanari M, Moja L, Apolone G. Prevalence of undertreatment in cancer pain. A review of published literature. Ann Oncol. 2008 Dec;19(12):1985-91.

4. Heading G, Mallock N, Sinclair S, Bishop J. New South Wales cancer patient satisfaction survey. Interim report. Sydney: Cancer Institute, NSW; 2008.

5. Benson M. NICS-VQC Fellow 2006-2008, Project: Improving the management of cancer pain2008.

6. Brink-Huis A, van Achterberg T, Schoonhoven L, Brink-Huis A, van Achterberg T,

Schoonhoven L. Pain management: a review of organisation models with integrated processes for the management of pain in adult cancer patients. J Clin Nurs. 2008 Aug;17(15):1986-2000.

7. Davies P, Walker AE, Grimshaw JM. A systematic review of the use of theory in the design of guideline dissemination and implementation strategies and interpretation of the results of rigorous evaluations. Implement Sci. 2010;5:14.

8. National Pain Summit Initiative. National Pain Strategy. Melbourne: Faculty of Pain Medicine2010.

9. Lovell M, Agar M, Luckett T, Davidson P, Green A, Clayton J. Australian survey of current practice and guideline use in adult cancer pain assessment and management: Perspectives of palliative care physicians. Journal of Palliative Medicine. 2014;10(2):e99-107. doi: 10.1111/ajco.12040.

10. Luckett T, Davidson PM, Boyle F, Liauw W, Agar M, Green A, et al. Australian survey of current practice and guideline use in adult cancer pain assessment and management: Perspectives of oncologists. Asia Pacific Journal of Clinical Oncology. 2012 doi: 10.1111/ajco.12040.

11. Phillips J, Lovell M, Luckett T, Agar M, Green A, Davidson P. Australian survey of current practice and guideline use in adult cancer pain assessment and management: The community nurse perspective. Collegian. 2014:DOI: http://dx.doi.org/10.1016/j.colegn.2013.11.002.

12. The ADAPTE Collaboration. The ADAPTE process: Resource toolkit for guideline adaptation. Version 2.0. Available from www.g-i-n.net2009.

13. Chakraborty SP, Jones KM, Mazza D. Adapting lung cancer symptom investigation and referral guidelines for general practitioners in Australia: Reflections on the utility of the ADAPTE framework. J Eval Clin Pract. 2014 Apr;20(2):129-35.

14. Cooley ME, Lobach DF, Johns E, Halpenny B, Saunders TA, Del Fiol G, et al. Creating computable algorithms for symptom management in an outpatient thoracic oncology setting. $J$ Pain Symptom Manage. 2013 Dec;46(6):911-24.e1.

15. Macintyre PE, Schug SA, Scott DA, Visser EJ, Walker SM, APM:SE Working Group of the Australian and New Zealand College of Anaesthetists and Faculty of Pain Medicine. Acute Pain Management: Scientific Evidence. Melbourne: ANZCA \& FPM; 2010.

16. Green E, Zwaal C, Beals C, Fitzgerald B, Harle I, Jones J, et al. Cancer-related pain management: a report of evidence-based recommendations to guide practice. Clin J Pain. $2010 \mathrm{Jul}-$ Aug;26(6):449-62. 
17. Portenoy R. Treatment of cancer pain. Lancet. 2011;377:2236-47.

18. Pigni A, Brunelli C, Gibbins J, Hanks G, Deconno F, Kaasa S, et al. Content development for European guidelines on the use of opioids for cancer pain: a systematic review and Expert Consensus Study. Minerva Anestesiologica. 2010 Oct;76(10):833-43.

19. Fervers B, Burgers JS, Voellinger R, Brouwers M, Browman GP, Graham ID, et al. Guideline adaptation: an approach to enhance efficiency in guideline development and improve utilisation. BMJ Qual Saf. 2011 Mar;20(3):228-36.

20. Breast Cancer Centre and National Cancer Control Initiative. Clinical practice guidelines for the psychosocial care of adults with cancer. Camperdown, Australia: National Breast Cancer Centre2003.

21. Luckett T, Davidson PM, Green A, Boyle F, Stubbs J, Lovell M. Assessment and management of adult cancer pain: a systematic review and synthesis of recent qualitative studies aimed at developing insights for managing barriers and optimizing facilitators within a comprehensive framework of patient care. Journal of Pain and Symptom Management. 2013;46(2):229-53.

22. Marie N, Luckett T, Davidson PM, Lovell M, Lal S. Optimal patient education for cancer pain : A systematic review and theory-based meta-analysis. Journal of Supportive Care in Cancer. 2013;21(12):3529-37.

23. Improving Palliative Care through Clinical Trials (ImpaCCT). Cancer pain management in adults: Evidence-based clinical practice guidelines adapted for use in Australia. 2013; Available from: http://wiki.cancer.org.au/australia/Guidelines:Cancer_pain_management.

24. National Health and Medical Research Council. NHMRC standards and procedures for externally developed guidelines. Canberra: NHMRC2007.

25. NICE clinical guideline 140. Opioids in palliative care: safe and effective prescribing of strong opioids for pain in palliative care of adults. London: NICE2012.

26. Caraceni A, Hanks G, Kaasa S, Bennett MI, Brunelli C, Cherny N, et al. Use of opioid analgesics in the treatment of cancer pain: evidence-based recommendations from the EAPC. Lancet Oncol. 2012 Feb;13(2):e58-68.

27. Scottish Intercollegiate Network (SIGN). Control of Pain in Patients with Cancer: A national clinical guideline. Edinburgh2008. Report No.: 106.

28. NHS Quality Improvement Scotland. Best practice statement: The management of pain in patients with cancer. Edinburgh: NHS Quality Improvement Scotland2004.

29. National Comprehensive Cancer Network (NCCN). NCCN Clinical Practice Guidelines in Oncology: Adult cancer pain. Version 2.2011. New York: NCCN2011.

30. Swarm RA, Abernethy AP, Anghelescu DL, Benedetti C, Buga S, Cleeland C, et al. Adult cancer pain. Journal of the National Comprehensive Cancer Network.11(8):992-1022.

31. Ripamonti CI, Bandieri E, Roila F, Group EGW. Management of cancer pain: ESMO Clinical Practice Guidelines. Ann Oncol. 2011 Sep;22 Suppl 6:vi69-77.

32. Roxburgh A, Bruno R, Larance B, Burns L. Prescription of opioid analgesics and related harms in Australia. Med J Aust. 2011 Sep 5;195(5):280-4.

33. Oldenmenger WH, Sillevis Smitt PAE, van Dooren S, Stoter G, van der Rijt CCD. A systematic review on barriers hindering adequate cancer pain management and interventions to reduce them: a critical appraisal. Eur J Cancer. 2009 May;45(8):1370-80.

34. World Health Organization. Cancer pain relief. Geneva: WHO1996. 
35. Klepstad P1, Kaasa S, Borchgrevink PC. Starting Step III opioids for moderate to severe pain in cancer patients: Dose titration: A systematic review. Palliative Medicine. 2011;25:424-30.

36. Dy SM, Asch SM, Naeim A, et al. Evidence-based recommendations for cancer pain management. J Clin Oncol. 2008;26:3879-85.

37. Olver IN, Von Dincklage JJ. It is time for clinical guidelines to enter the digital age. Med J Aust. 2013 Nov 4;199(9):569-70.

38. Cummings GG, Olivo SA, Biondo PD, Stiles CR, Yurtseven O, Fainsinger RL, et al. Effectiveness of knowledge translation interventions to improve cancer pain management. J Pain Symptom Manage. 2011 May;41(5):915-39.

39. Ivers N, et al. Audit and feedback: effects on professional practice and patient outcomes. Cochrane Database of Systematic Reviews. 2012;Published online July 11th.

40. Cancer Council Australia. Overcoming cancer pain: A guide for people with cancer, their families and friends. 2013 [cited 2014 18th February]; Available from: http://www.cancercouncil.com.au/wp-content/uploads/2014/01/Can487-Overcoming-Pain-NSWLores2.pdf.

41. Lovell MR, Forder P, Stockler M, Butow PN, Briganti E, Chye R, et al. A randomised controlled trial of a standardised educational intervention for patients with cancer pain. Journal of Pain and Symptom Management. 2010;40(1):49-59.

42. Kerfoot BP, Kearney MC, Connelly D, Ritchey ML. Interactive spaced education to assess and improve knowledge of clinical practice guidelines: a randomized controlled trial. Ann Surg. 2009 May;249(5):744-9.

43. Brando A, editor. Transforming literature findings to practical indicators - an effort to incorporate best practice pain management for palliative care patients. abs\#418. Annual Scientific Meeting of the Clinical Oncological Society of Australia (COSA); 2011; Perth: Asia-Pacific Journal of Clinical Oncology. 